TecnoLógicas

ISSN-p 0123-7799

ISSN-e 2256-5337

Vol. 21, No. 43, pp. 43-52

Sep-dic de 2018

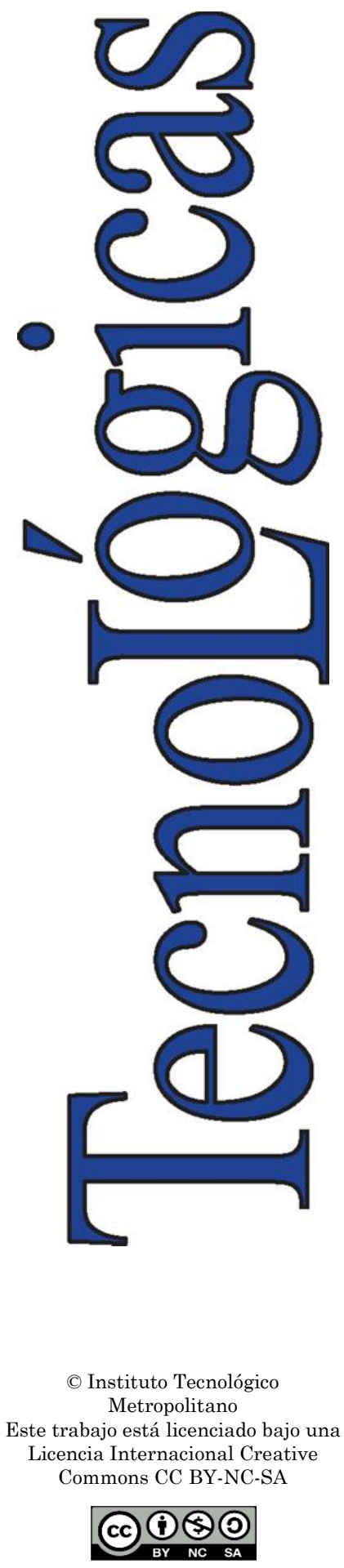

Artículo de Investigación/Research Article

\section{First principles calculations of the electronic and dielectric properties of $\lambda$-Ta $\mathbf{a}_{2}$}

\author{
Cálculo de primeros principios de las \\ propiedades electrónicas y dieléctricas \\ de $\lambda-\operatorname{Ta}_{2} \mathbf{0}_{5}$
}

\author{
Camilo Valencia-Balvín¹, Santiago Pérez-Walton², y \\ Jorge M. Osorio-Guillén ${ }^{3}$
}

Recibido: 14 de junio de 2018

Aceptado: 29 de agosto de 2018

Cómo citar / How to cite

C. Valencia-Balvín, S. Pérez-Walton, y Jorge M. Osorio-Guillén, First principles calculations of the electronic and dielectric properties of $\lambda$ Ta2 $\mathrm{O}_{5}$. TecnoLógicas, vol. 21, no. 43, pp. 43-52, 2018.

1 PhD. en Física, Departamento de Electrónica y Telecomunicaciones, Facultad de Ingenierías, Instituto Tecnológico Metropolitano, MedellínColombia, camilovalencia@itm.edu.co

2 PhD. en Física, Departamento de Electrónica y Telecomunicaciones, Facultad de Ingenierías, Instituto Tecnológico Metropolitano, MedellínColombia, santiagoperez@itm.edu.co

3 PhD. en Física, Instituto de Física, Universidad de Antioquia, MedellínColombia, mario.osorio@udea.edu.co 


\section{Abstract}

$\mathrm{Ta}_{2} \mathrm{O}_{5}$ is a wide-bandgap semiconductor that offers interesting applications in microwave communications, mainly related to the manufacture of filters and resonators whose size is inversely proportional to the dielectric constant of the material. For that reason, in this work we present a theoretical study, based on density functional theory (using PBEsol and hybrid HSE06 exchange-correlation functionals), of the electronic and dielectric properties of the orthorhombic model $\lambda-\mathrm{Ta}_{2} \mathrm{O}_{5}$. We found that this model has a direct gap of 2.09 and $3.7 \mathrm{eV}$ with PBEsol and HSE06, respectively. Furthermore, the calculated static dielectric constant, 51, is in good agreement with the reported values of other phases of this semiconductor.

\section{Keywords}

$\mathrm{Ta}_{2} \mathrm{O}_{5}$, density functional theory, PBEsol, HSE06, dielectric constant.

\section{Resumen}

$\mathrm{Ta}_{2} \mathrm{O}_{5}$ es un semiconductor de gap-ancho el cual tiene interesantes aplicaciones en comunicaciones en la región de las micro-ondas, principalmente está relacionado con la fabricación de filtros y resonadores, donde su tamaño es inversamente proporcional a la constante dieléctrica del material. Por este motivo, en este trabajo presentamos un estudio teórico a partir de la teoría de los funcionales de la densidad (usando PBEsol y el híbrido HSE06 para el funcional de intercambio-correlación) de las propiedades electrónicas y dieléctricas del modelo ortorrómbico $\lambda-\mathrm{Ta}_{2} \mathrm{O}_{5}$. Aquí, encontramos que este modelo tiene un gap directo de 2.09 y $3.7 \mathrm{eV}$ con PBEsol y HSE06, respectivamente. Además, la constante dieléctrica estática calculada es 51, en buen acuerdo con los valores reportados para otras fases de este semiconductor.

\section{Palabras clave}

$\mathrm{Ta}_{2} \mathrm{O}_{5}$, teoría de los funcionales de la densidad, PBEsol, HSE06, constante dieléctrica. 


\section{INTRODUCTION}

For many years, semiconductors have been used in a wide variety of applications that have allowed developments, at a fairly accelerated rate, in several fields of science and engineering. In this context, widebandgap semiconductors have drawn great interest in recent years due to their applications in some areas of knowledge, such as electronics, communications and energy [1]-[3]. One of the semiconductors that offers great potential for this type of applications is $\mathrm{Ta}_{2} \mathrm{O}_{5}$. Its properties include high dielectric constant, low dielectric loss and good temperature stability. As a result, it can be used, for instance, as a dielectric resonator in microwave communication, where miniaturization critically depends on the dielectric constant of the ceramic component of the device. This indicates that a large dielectric constant, in principle, means a small size for the resonator [2].

$\mathrm{Ta}_{2} \mathrm{O}_{5}$ has a set of crystallographic phases that depend on temperature and pressure. To describe some of these phases, there are experimental models which can adequately explain the different chemical and physical properties associated with this compound [4]. Among all the known phases of this semiconductor, the modification of $\mathrm{L}_{-} \mathrm{Ta}_{2} \mathrm{O}_{5}$ without oxygen vacancies, reported in different experimental and theoretical studies, awakens important interest [5]-[9]. Lee et al. [10] proposed a model to describe it based on its orthorhombic structure with two formula unit per unit cell, called $\lambda$ phase, where its local structure corresponds to chains of distorted octahedral $\mathrm{TaO}_{6}$. In this work, we have adopted such model to study the structural, electronic and optical properties of the low-temperature modification. We indirectly compared our results with experimental and theoretical values reported in other studies.

\section{COMPUTATIONAL METHODS}

\subsection{Crystal and electronic structure calculations}

To study the physical properties of $\mathrm{Ta}_{2} \mathrm{O}_{5}$, we calculated the total energy as a function of the volume of the unit cell, completely relaxing the ionic positions and the shape of the unit cell for each one of the calculated volumes. Afterward, the obtained data were adjusted to the fourthorder Birch-Murnaghan equation of state [11], [12] and, based on it, we identified the equilibrium geometry [4]. Total energy, the forces on the ions and the components of the stress tensor in this work were calculated using density functional theory (DFT). For the exchange-correlation functional, we used the generalized gradient approximation (GGA) with PBEsol parametrization [13] and the hybrid functional HSE06 [14], [15]. The Kohn-Sham equations were solved using the projector augmented plane-wave (PAW) method [16] as implemented in the VASP code [17], [18]. The reference PAW atomic configurations were: $5 p^{6} 5 d^{4} 6 s^{1}$ for Ta and $2 s^{2} 2 p^{4}$ for $\mathrm{O}$, where only states that are treated as valence electrons were listed. The cut-off energy in the plane-wave expansion was converged to a value of 520 $\mathrm{eV}$.

All the structural parameters for each one of the calculated volumes were optimized, by simultaneously minimizing the atomic forces and the components of the stress tensor, through the conjugate gradient algorithm. The integration in the first Brillouin zone was carried out using a $4 \times 4 \times 8$ Monkhorst-Pack $\mathbf{k}$-mesh with a Gaussian broadening of $0.01 \mathrm{eV}$ for relaxation (the original forces were converged to $1 \mathrm{meV} / \AA ̊$ ). Subsequently, a calculation was made at a $4 \times 4 \times 8 \quad \Gamma$ centered $\mathbf{k}$-mesh for the total energy (the energy was converged to $1 \mathrm{meV} /$ unit cell), the charge density, and the dielectric and optical properties. 


\subsection{Calculation of dielectric and optical properties}

We calculated the static dielectric constant $\varepsilon(0)$ using density functional perturbation theory (DFPT) [19], where (1) is the Sternheimer linear equation, was first solved for $\left|\nabla_{\boldsymbol{k}} \tilde{u}_{n \boldsymbol{k}}\right\rangle$, where $\tilde{u}_{n \boldsymbol{k}}$ is the periodic part of the auxiliary wave function; $\boldsymbol{H}(\boldsymbol{k})$, the effective Hamiltonian of an electron; $\epsilon_{n k}$, the Kohn-Sham energy; $n$, the band; $\mathbf{k}$, the Bloch wave vector; and $\boldsymbol{S}(\boldsymbol{k})$, the overlapping operator. Once this equation was solved, we determined the first-order response of the wave functions $\left|\zeta_{n \boldsymbol{k}}\right\rangle$ to an externally applied field by solving the linear equation (2), where $\Delta \boldsymbol{H}_{S C F}(\boldsymbol{k})$ represents the microscopic optic change of the Hamiltonian due to the variation of the wave functions to firstorder, $\widehat{\boldsymbol{q}}$ corresponds to the principal axes of the macroscopic dielectric matrix, and $\vec{\beta}_{n \boldsymbol{k}}$ is the polarization vector. Finally, with the information obtained by solving (1) and (2), the static dielectric constant can be determined using (3), where $\Omega$ is the volume of the primitive cell and $\omega_{\boldsymbol{k}}$ are the $\mathrm{k}$-point weights, which are defined in such way that their sum equals 1 . The optical properties associated with inter-band transitions was determined from the calculation of the imaginary part of the dielectric tensor $\left(\varepsilon^{(2)}(\hat{q}, \omega)\right)$ at the long wavelength limit $(\vec{q} \rightarrow 0)$ using the methodology developed for the PAW method [19]. The real part of the frequency-dependent dielectric tensor is obtained from the imaginary part through the Kramers-Kröning relationship. From the knowledge of the dielectric tensor, we have calculated the generalized refractive index $(\tilde{n}=n+i \kappa)$ and the transmittance explicitly using the equations in [20]. We did not include the spin-orbit coupling (SOC) because the Tantalum atom, which is the chemical species that introduces relativistic effects, mostly affects the conduction bands. The main contribution to the dielectric function is due to the ionic part, which is obtained using the Sternheimer equation, where the occupied bands are the only ones to be considered in this calculation. As a result, the SOC becomes non-significant to estimate the dielectric function in this case.

\section{RESULTS AND DISCUSSION}

\subsection{Crystal structure}

$\lambda-\mathrm{Ta}_{2} \mathrm{O}_{5}$ is an orthorhombic model described by the space group Pbam with two formula unit per unit cell, where four $\mathrm{Ta}$ atoms are located in the Wyckoff position (4g), while ten $\mathrm{O}$ atoms are distributed over the Wyckoff positions (2a), $(4 \mathrm{~g})$ and $(4 \mathrm{~h})$. It has a crystal structure is build up for a set of $\mathrm{TaO}_{6}$ octahedra where the Ta atom is located approximately in the center of the octahedra and the $\mathrm{O}$ atoms, in the corners. In addition, consecutive octahedra form chains that share the corners, as illustrated in Fig 1.

$$
\begin{gathered}
{\left[\boldsymbol{H}(\boldsymbol{k})-\epsilon_{n \boldsymbol{k}} \boldsymbol{S}(\boldsymbol{k})\right]\left|\boldsymbol{\nabla}_{\boldsymbol{k}} \tilde{u}_{n \boldsymbol{k}}\right\rangle=-\frac{\partial\left[\boldsymbol{H}(\boldsymbol{k})-\epsilon_{n \boldsymbol{k}} \boldsymbol{S}(\boldsymbol{k})\right]}{\partial \boldsymbol{k}}\left|\tilde{u}_{n \boldsymbol{k}}\right\rangle} \\
{\left[\boldsymbol{H}(\boldsymbol{k})-\epsilon_{n \boldsymbol{k}} \boldsymbol{S}(\boldsymbol{k})\right]\left|\zeta_{n \boldsymbol{k}}\right\rangle=-\Delta \boldsymbol{H}_{S C F}(\boldsymbol{k})\left|\tilde{u}_{n \boldsymbol{k}}\right\rangle-\left|\widehat{\boldsymbol{q}} \vec{\beta}_{n \boldsymbol{k}}\right\rangle} \\
\varepsilon_{\infty}(\widehat{\boldsymbol{q}}, 0)=1-\frac{8 \pi e^{2}}{\Omega} \sum_{v, \boldsymbol{k}} 2 \omega_{\boldsymbol{k}}\left\langle\widehat{\boldsymbol{q}}_{v \boldsymbol{k}} \mid \zeta_{v \boldsymbol{k}}\right\rangle
\end{gathered}
$$




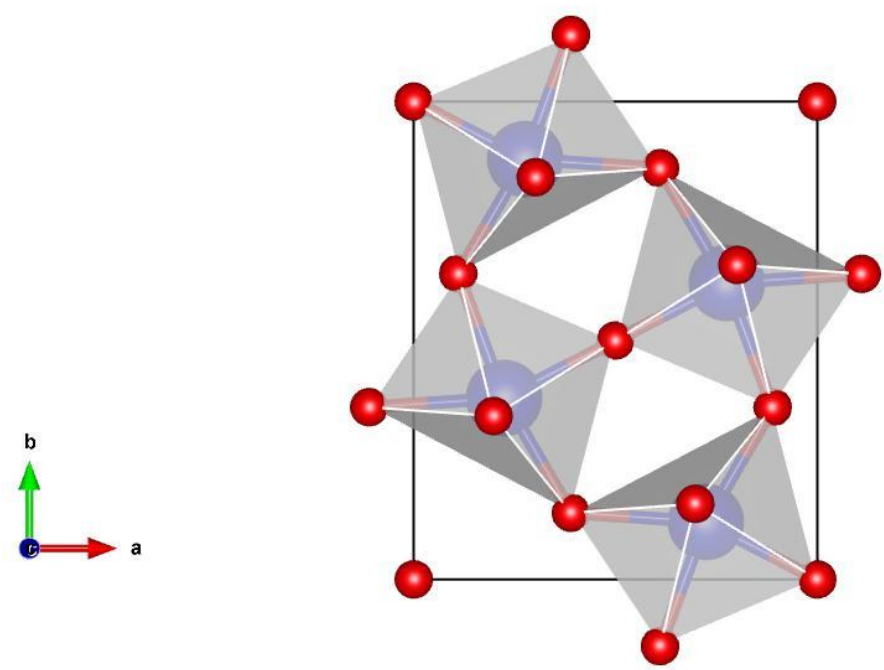

Fig. 1. Crystal structure of $\lambda-\mathrm{Ta}_{2} \mathrm{O}_{5}$. Purple and red circles represent Ta and $\mathrm{O}$ atoms, respectively. Source: Authors' own work.

At this point, it is very important to mention that the orthorhombic symmetry was preserved during the relaxation process for all the fixed volumes, which allowed us to find the equilibrium geometry. Table 1 presents the most important crystallographic parameters associated with the $\lambda$ model. Clearly, our results of the calculation with both functionals are in very good agreement with the optimized parameters in the model by Lee et al.

\subsection{Electronic structure}

The calculated dispersion relation for the $\lambda$ model is presented in Fig 2 . The gap of this orthorhombic model calculated with PBEsol and HSE06 was $2.09 \mathrm{eV}$ and 3.7 $\mathrm{eV}$, respectively, where the HSE06 prediction is in good agreement with the value reported by Lee et al. [10] and the experimental gap reported for the $\mathrm{L}$ phase $(4.0 \mathrm{eV})[21]$. This is because excited state properties are better predicted by explicitly orbital-dependent functionals, which include exact exchange such as HSE06. On the other hand, we found that $\lambda-\mathrm{Ta}_{2} \mathrm{O}_{5}$ has a direct energy band gap, where the valence band maximum (VBM) and conduction band minimum (CBM) are located in the high symmetry point $\mathrm{Z}=(0$, $0,1 / 2)$.

A detailed analysis of the band structure allowed us to determine that the valence band (VB) is a hybridization of Ta $d$-states and $\mathrm{O} p$-states, where the latter present the greatest contribution to VBM. On the other hand, the CBM is dominated by Ta $d$-states. Finally, we can observe from Fig. 2 that HSE06 produces a rigid band shift of the $\mathrm{CB}$ to higher energies with respect to VBM, while the VB presents a slightly larger width compared to PBEsol. The topology of the band structure is basically the same for both functionals.

Table 1. Calculated and experimental crystallographic parameters of $\lambda-\mathrm{Ta}_{2} \mathrm{O}_{5}$. The model in this work represents the values

\begin{tabular}{lllll}
\multicolumn{5}{c}{ given in [10]. Source: Authors' own work and [10]. } \\
\hline & $\boldsymbol{a}(\AA)$ & $\boldsymbol{b}(\AA)$ & $\boldsymbol{c}(\AA)$ & $\boldsymbol{\Omega}\left(\AA^{\mathbf{3}}\right)$ \\
\hline PBEsol & 6.197 & 7.333 & 3.793 & 172.36 \\
HSE06 & 6.201 & 7.333 & 3.791 & 172.38 \\
Model & 6.25 & 7.4 & 3.83 & 177.14 \\
\hline
\end{tabular}



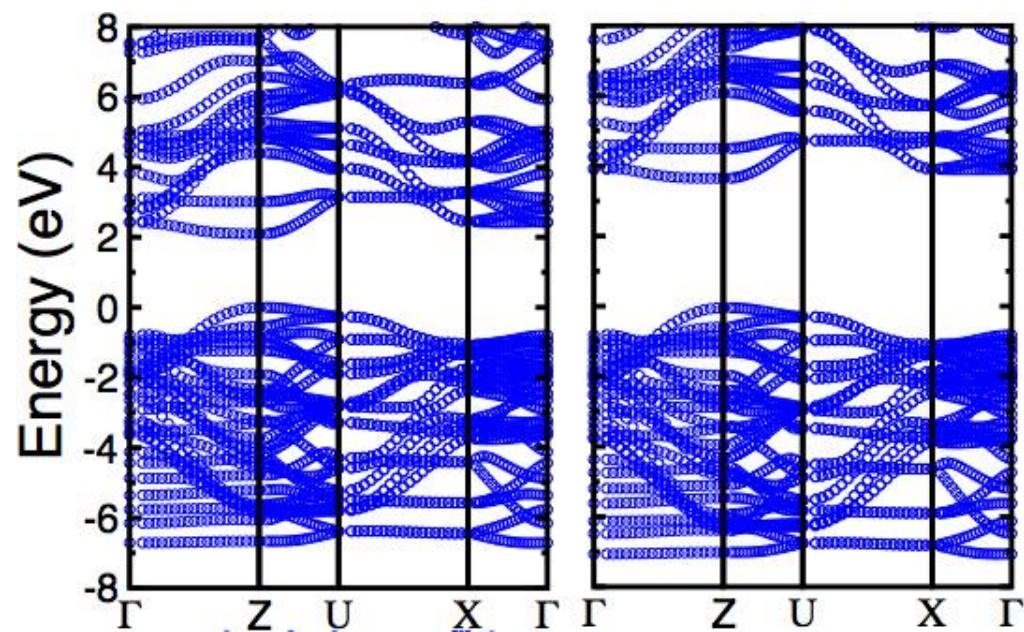

Fig. 2. Band structure of $\lambda-\mathrm{Ta}_{2} \mathrm{O}_{5}$. Left: PBEsol eigenvalues. Right: HSE06 eigenvalues. Source: Authors' own work.

\subsection{Dielectric tensor}

The dielectric function is second rank tensor, for an orthorhombic crystal it only has three components different from zero: $\left(\varepsilon_{x x}, \varepsilon_{y y}, \varepsilon_{z z}\right)$. Fig. 3 (a-c) shows the diagonal components of the imaginary part of the dielectric tensor $\left(\varepsilon_{\alpha \beta}^{(2)}\right)$ calculated with PBEsol and HSE06 functionals. In this case, it is easy to identify some degree of anisotropy in the dielectric tensor. This situation can be observed from the position of the main absorption peaks among its components, which are slightly located at different energies. Furthermore, the intensity of the main peak of the $\varepsilon_{z z}$ component is greater than the other two components, which have fairly close values. Here, it is important to mention that the main peaks in Fig. 3 are associated with inter-band transitions between $\mathrm{O} p$-states in the VB and Ta $d$ states in the conduction band.

Table 2 presents the calculated electronic $\left(\varepsilon_{\infty}\right)$ and ionic contribution $\left(\varepsilon_{0}\right)$ to the static dielectric tensor with PBEsol parametrization. Using these results, we were able to calculate the directional average and obtain the static dielectric constant $\left(\varepsilon(0)=\varepsilon_{\infty}+\varepsilon_{0}\right)$, which equals 51 . In this case, our results cannot be directly compared with experimental data, since the experimental value for the static dielectric constant of this type of structures has not been reported. However, we can make an indirect comparison with the calculation performed by Clima et al. [22] for the orthorhombic phase proposed by Ramprasad [7], which reports the following values for the contributions to the static dielectric constant: $\varepsilon_{\infty}=5.92$ and $\varepsilon_{0}=36$. By comparing these values with our calculation, we find that the $\varepsilon_{\infty}$ component is in good agreement with the value reported by Clima et al.; however, our calculated $\varepsilon_{0}$ is much higher than in the model described by said authors. This is because the ionic contribution of the dielectric constant is strongly dependent on the crystallographic system. Therefore, an orthorhombic model composed of pentagonal bipyramids and distorted octahedra Clima et al.) is very different from a Lambda model defined by distorted octahedra only. 


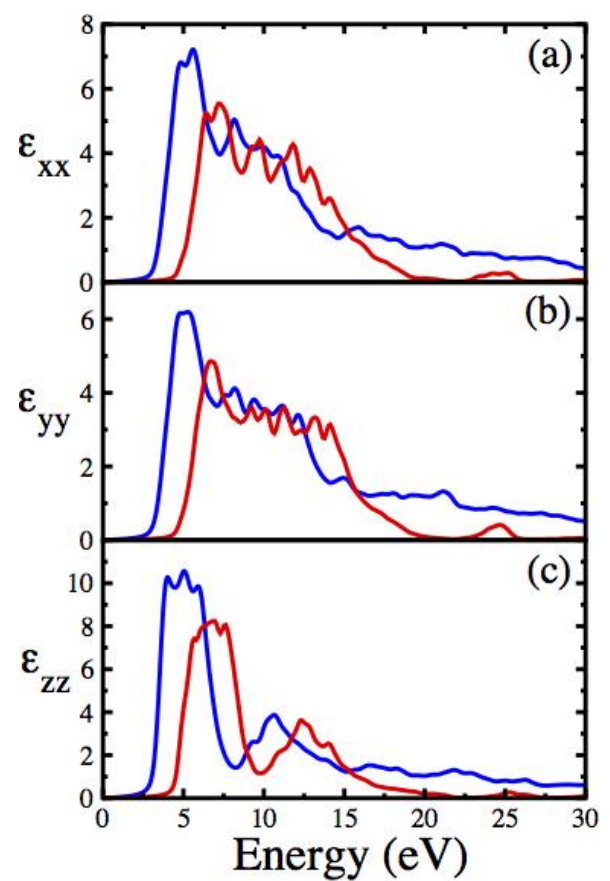

Fig. 3. Non-zero components of the imaginary part of dielectric tensor of $\lambda-\mathrm{Ta}_{2} \mathrm{O}_{5}$. Blue and red lines represent PBEsol and HSE06 calculations, respectively. Source: Authors' own work.

Table 2. Calculated optical-frequency and low-frequency dielectric tensor for $\lambda$-Ta $\mathrm{Ta}_{2} \mathrm{O}_{5}$ with PBEsol parametrization. Source: Authors' own work.

$$
\varepsilon_{\infty}=\left(\begin{array}{ccc}
\mathbf{5 . 8 2 3} & \mathbf{0} & \mathbf{0} \\
\mathbf{0} & \mathbf{5 . 4 5 1} & \mathbf{0} \\
\mathbf{0} & 0 & 7.290
\end{array}\right)
$$

$\varepsilon_{0}$
$=\left(\begin{array}{ccc}45.636 & 0 & 0 \\ 0 & 38.814 & 0 \\ 0 & 0 & 49.762\end{array}\right)$

\subsection{Optical properties}

Once the dielectric tensor was calculated along the three Cartesian directions, we obtained the generalized refractive index $(\tilde{n})$. Fig. 4 shows the refractive index $n$ and the extinction coefficient $\kappa$. It can be seen from this figure that the value calculated with PBEsol (HSE06) for $n$ is 2.48 (2.02). In the literature, we did not find a reported value of refractive index for this model. However, as in the case of phase B, we can make an indirect comparison with the value reported for an ultra-thin film of $\mathrm{Ta}_{2} \mathrm{O}_{5}(n$ $=2.15$ ) [23], which is consistent with our calculation. In addition, the extinction coefficient enabled us to observe maximum absorption peaks at $6.1 \mathrm{eV}$ and $11.3 \mathrm{eV}(7.8$ $\mathrm{eV}$ and $14.4 \mathrm{eV}$ ) with PBESol (HSE06), which are in the region of strong absorption within the ultraviolet region.

Finally, we calculated the transmittance (Fig 5). From this figure it can be observed that, in the visible region (between 0.1 and $1 \mu \mathrm{m}$ ), the behavior of the transmittance is constant with a value of $82 \%(88 \%)$ with PBESol (HSE06). This result allows us to conclude that, in this region, $\quad \lambda-\mathrm{Ta}_{2} \mathrm{O}_{5}$ is a transparent semiconductor. 


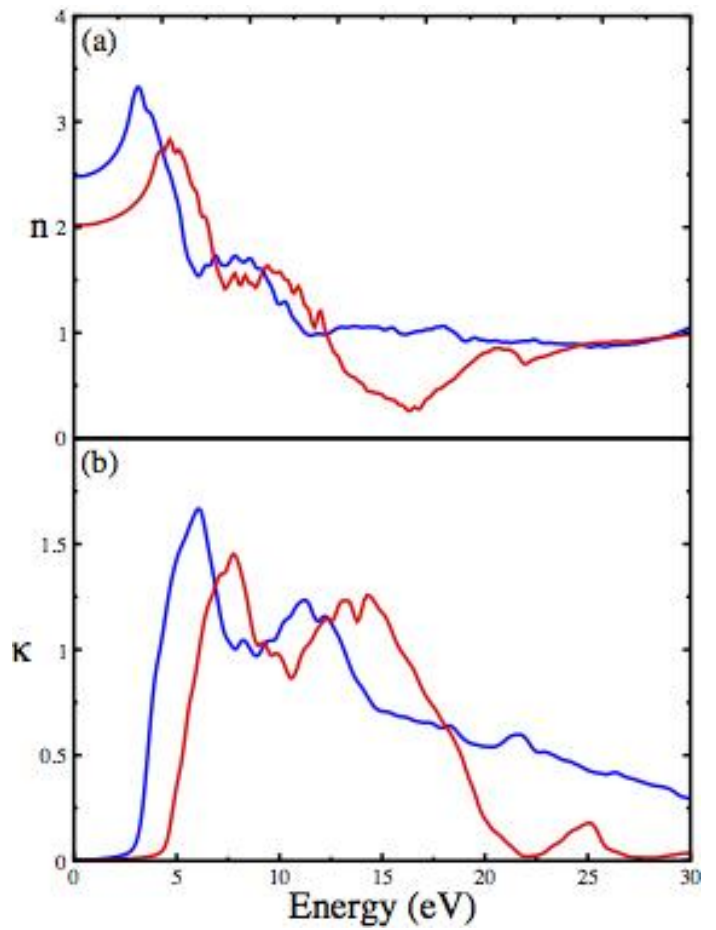

Fig. 4. a) Refractive index and b) extinction coefficient. Blue and red lines represent PBEsol and HSE06 calculations, respectively. Source: Authors' own work.

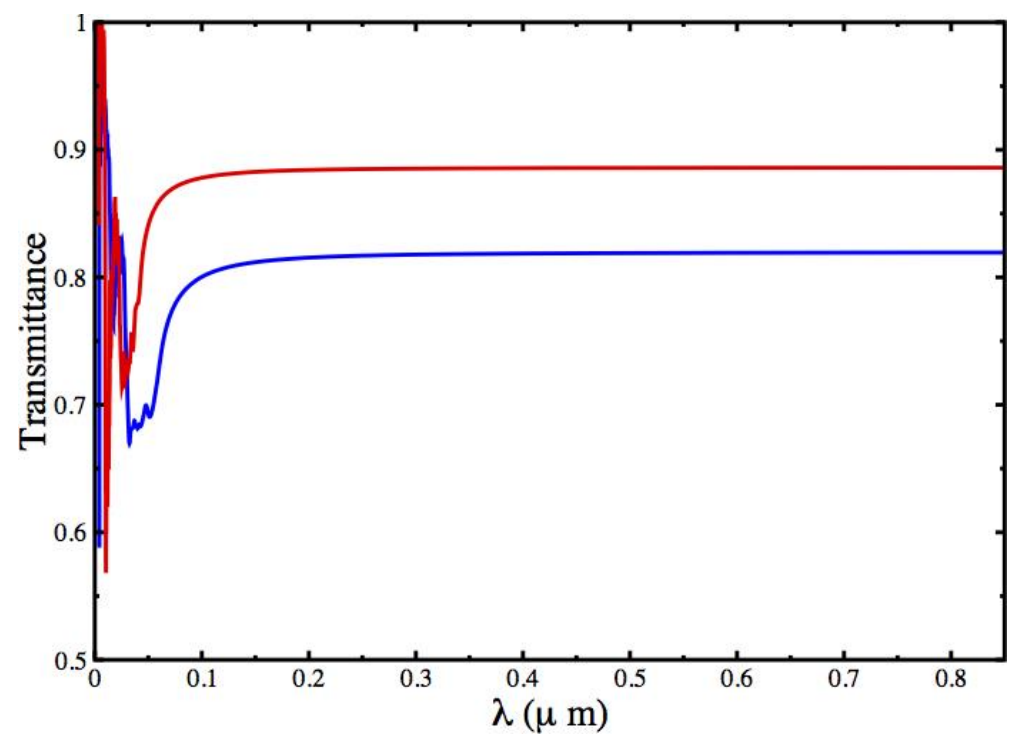

Fig. 5. Transmittance in the visible region. Blue and red lines represent PBEsol and HSE06 calculations, respectively. Source: Authors' own work.

\section{CONCLUSIONS}

In this work, we studied different physical properties of an orthorhombic $\lambda$ $\mathrm{Ta}_{2} \mathrm{O}_{5}$ model without oxygen vacancies. Based on our calculations, we were able to conclude that the VB is a hybridization of $\mathrm{O} p$ and $\mathrm{Ta} d$-states, while a large percentage of the $\mathrm{CB}$ is composed of Ta $d$ states. Furthermore, under this model $\mathrm{Ta}_{2} \mathrm{O}_{5}$ presents a direct gap, where VBM and CBM are located in a high symmetry point $\mathrm{Z}=(0,0,1 / 2)$. In addition, we found that the static dielectric constant, 51 , is in good agreement with values reported in other works. As a result, this novel 
First principles calculations of the electronic and dielectric properties of $\lambda-\mathrm{Ta}_{2} \mathrm{O}_{5}$

material can be used to manufacture filters and resonators. Finally, we calculated the transmittance and stablished that, in the visible region, this semiconductor described by the $\lambda$ model is a transparent material.

\section{ACKNOWLEDGMENTS}

The computations were performed on Cluster provided by Instituto Tecnológico Metropolitano (ITM). C. Valencia-Balvín. and S. Pérez-Walton acknowledge the Faculty of Engineering at ITM. Jorge Osorio-Guillén thanks Universidad de Antioquia.

\section{REFERENCES}

[1] M.-J. Lee et al., "A fast, high-endurance and scalable non-volatile memory device made from asymmetric $\mathrm{Ta}_{2} \mathrm{O}_{5}-\mathrm{x} / \mathrm{Ta}_{2} \mathrm{O}_{5}-\mathrm{x}$ bilayer structures," Nat. Mater., vol. 10, no. 8, pp. 625-630, Aug. 2011.

[2] R. J. Cava, "Dielectric materials for applications in microwave communications," J. Mater. Chem., vol. 11, no. 1, pp. 54-62, 2001.

[3] K. J. Kumar, N. R. C. Raju, and A. Subrahmanyam, "Properties of pulsed reactive DC magnetron sputtered tantalum oxide $\left(\mathrm{Ta}_{2} \mathrm{O}_{5}\right)$ thin films for photocatalysis," Surf. Coatings Technol., vol. 205, pp. S261S264, Jul. 2011.

[4] S. Pérez-Walton, C. Valencia-Balvín, A. C. M. Padilha, G. M. Dalpian, and J. M. OsorioGuillén, "A search for the ground state structure and the phase stability of tantalum pentoxide," J. Phys. Condens. Matter, vol. 28, no. 3, p. 035801, Jan. 2016.

[5] L. A. Aleshina and S. V Loginova, "Rietveld analysis of X-ray diffraction pattern from B$\mathrm{Ta}_{2} \mathrm{O}_{5}$ oxide," Crystallogr. Reports, vol. 47, no. 3, pp. 415-419, May 2002

[6] A. Fukumoto and K. Miwa, "Prediction of hexagonal $\mathrm{Ta}_{2} \mathrm{O}_{5}$ structure by first-principles calculations," Phys. Rev. B, vol. 55, no. 17, pp. 11155-11160, May 1997.

[7] R. Ramprasad, "First principles study of oxygen vacancy defects in tantalum pentoxide," J. Appl. Phys., vol. 94, no. 9, pp. 5609-5612, Nov. 2003.

[8] R. Nashed, W. M. I. Hassan, Y. Ismail, and N. K. Allam, "Unravelling the interplay of crystal structure and electronic band structure of tantalum oxide $\left(\mathrm{Ta}_{2} \mathrm{O}_{5}\right)$, , Phys. Chem. Chem. Phys., vol. 15, no. 5, pp. 13521357, 2013.

[9] W. Andreoni and C. A. Pignedoli, " $\mathrm{Ta}_{2} \mathrm{O}_{5}$ polymorphs: Structural motifs and dielectric constant from first principles," Appl. Phys. Lett., vol. 96, no. 6, p. 062901, Feb. 2010.

[10] S. H. Lee, J. Kim, S.-J. Kim, S. Kim, and G.S. Park, "Hidden Structural Order in Orthorhombic $\mathrm{Ta}_{2} \mathrm{O}_{5}$," Phys. Rev. Lett., vol. 110, no. 23, p. 235502, Jun. 2013.

[11] A. Otero-de-la-Roza and V. Luaña, "Gibbs2: A new version of the quasi-harmonic model code. I. Robust treatment of the static data," Comput. Phys. Commun., vol. 182, no. 8, pp. 1708-1720, Aug. 2011.

[12] A. Otero-de-la-Roza, D. Abbasi-Pérez, and V. Luaña, "Gibbs2: A new version of the quasiharmonic model code. II. Models for solid-state thermodynamics, features and implementation," Comput. Phys. Commun., vol. 182, no. 10, pp. 2232-2248, Oct. 2011.

[13] J. P. Perdew et al., "Restoring the DensityGradient Expansion for Exchange in Solids and Surfaces," Phys. Rev. Lett., vol. 100, no. 13, p. 136406, Apr. 2008.

[14] J. Heyd, G. E. Scuseria, and M. Ernzerhof, "Hybrid functionals based on a screened Coulomb potential," J. Chem. Phys., vol. 118, no. 18, pp. 8207-8215, May 2003.

[15] J. Heyd, G. E. Scuseria, and M. Ernzerhof, "Erratum: "Hybrid functionals based on a screened Coulomb potential," J. Chem. Phys., vol. 124, no. 21, p. 219906, Jun. 2006.

[16] P. E. Blöchl, "Projector augmented-wave method," Phys. Rev. B, vol. 50, no. 24, pp. 17953-17979, Dec. 1994.

[17] G. Kresse and D. Joubert, "From ultrasoft pseudopotentials to the projector augmentedwave method," Phys. Rev. B, vol. 59, no. 3, pp. 1758-1775, Jan. 1999.

[18] G. Kresse and J. Furthmüller, "Efficient iterative schemes for ab initio total-energy calculations using a plane-wave basis set," Phys. Rev. B, vol. 54, no. 16, pp. 1116911186, Oct. 1996.

[19] M. Gajdoš, K. Hummer, G. Kresse, J. Furthmüller, and F. Bechstedt, "Linear optical properties in the projector-augmented wave methodology," Phys. Rev. B, vol. 73, no. 4, p. 045112, Jan. 2006.

[20] S. Pérez-Walton, C. Valencia-Balvín, G. M. Dalpian, and J. M. Osorio-Guillén, "Electronic, dielectric, and optical properties of the $B$ phase of niobium pentoxide and tantalum pentoxide by first-principles calculations," Phys. status solidi, vol. 250, no. 8, pp. 1644-1650, Aug. 2013.

[21] I. E. Wachs, Y. Chen, J.-M. Jehng, L. E. 
Briand, and T. Tanaka, "Molecular structure and reactivity of the Group V metal oxides," Catal. Today, vol. 78, no. 1-4, pp. 13-24, Feb. 2003.

[22] S. Clima et al., "Dielectric Response of $\mathrm{Ta}_{2} \mathrm{O}_{5}$, $\mathrm{NbTaO}_{5}$ and $\mathrm{Nb}_{2} \mathrm{O}_{5}$ from First-Principles
Investigations," in ECS Transactions, 2009, vol. 19, pp. 729-737.

[23] D.-X. Zhang et al., "Thickness-dependence of optical constants for $\mathrm{Ta}_{2} \mathrm{O}_{5}$ ultrathin films," Appl. Phys. A, vol. 108, no. 4, pp. 975-979, Sep. 2012. 